ARTÍCULO ORIGINAL

\title{
Evaluación de la actividad física para alcanzar un estilo de vida activo en los profesionales de la salud: estudio piloto
}

Andrea Villegas-Narváez*, Luz Urbina-Arronte, Ana Cristina García-Ulloa y Sergio Hernández-Jiménez; Grupo de Trabajo CAIPaDi

Centro de Atención Integral del Paciente con Diabetes, Instituto Nacional de Ciencias Médicas Salvador Zubirán, Ciudad de México, México

\section{RESUMEN}

El objetivo de este estudio fue conocer el nivel de actividad física (AF) en profesionales de la salud (PS) mediante el uso de un podómetro para mantener un estilo de vida activo a 12 semanas. Para este estudio piloto se incluyeron 26 participantes. Se aplicó el Cuestionario Internacional de Actividad Física (IPAQ versión en español) y se asignó un monitor de AF a cada participante. Se realizó un análisis descriptivo de pasos y composición corporal.

Al inicio, el promedio de pasos era de 7,340. A las 12 semanas, éste se incrementó a 8,535 $(p=0.06)$. El peso disminuyó de 61.6 a $61.3 \mathrm{~kg}$ en 12 semanas. En relación con el índice de masa corporal (IMC), masa grasa (MBF) y masa muscular (SLM) no se observaron cambios significativos. Las barreras más frecuentes fueron falta de tiempo y tipo de trabajo. En este estudio, los PS no cumplieron con las

\section{ABSTRACT}

The aim of this study was to know the level of AF in health professionals (HP) by using a pedometer, to maintain an active lifestyle for 12 weeks. For this pilot study we included 26 participants. The international physical activity questionnaire was answered and a physical activity monitor was assigned to each participant. The number of steps per day was recorded each week and strategies were provided to increase AF. We did a descriptive analysis of steps and body composition. At the beginning, the average number of steps was 7,340. At 12 weeks it increased to $8,535(p=0.06)$. The weight decreased from $61.6 \mathrm{Kg}$ to $61.3 \mathrm{Kg}$ in 12 weeks. No significant changes were observed in relation of body mass index (BMI), mass body fat (MBF) and skeletal lean mass (SLM). The most common barrier was lack of time and type of work. The PS in this study did not reach with the AF
Correspondencia:

*Andrea Villegas-Narváez

E-mail: andreavillegasnardgmail.com
Fecha de recepción: 14-06-2018

Fecha de aceptación: 26-08-2018 Doi: 10.24875/ALAD.18000332 
recomendaciones de AF reportadas en las guías internacionales.

Palabras clave: Podómetro. Actividad física. Profesionales de la salud. recommendations reported in the international guidelines. (Rev ALAD. 2018;8:145-51)

Corresponding author: Andrea Villegas-Narváez andreavillegasnar@gmail.com

Key words: Pedometer. Physical activity. Health professionals.

\section{INTRODUCCIÓN}

La AF se define como cualquier movimiento del cuerpo que produce un gasto de energía, como caminar hacia el trabajo, labores domésticas, subir y bajar escaleras, etc. ${ }^{1}$. En la actualidad se utilizan podómetros como herramientas útiles para medir de forma objetiva la cantidad de AF, pues éstos cuantifican el número de pasos que se realizan cotidianamente ${ }^{2,3}$. En 2003, Tudor-Locke y Basset propusieron una clasificación basada en la cantidad de pasos al día, considerándose sedentario cuando se realizan menos de 5,000 pasos al día, nivel bajo de AF cuando se realizan 5,000-7,499 pasos al día, AF moderada cuando se realizan 7,500-9,999 pasos al día, AF alta cuando se dan 10,000-12,499 pasos al día y AF muy alta al alcanzar más de 12,500 pasos al día².

La Organización Mundial de la Salud recomienda que la AF mínima debe constar de 10,000 pasos al día; sin embargo, esta misma organización reporta que más del $60 \%$ de la población mundial no logra esa meta 4 .

El sedentarismo se ha definido como la situación en la que no hay AF (como estar sentado viendo la televisión, frente a la computadora, en trabajo de escritorio) por tiempo prolongado. Se ha reportado que algunas personas pueden pasar hasta $11 \mathrm{~h}$ del día en estas circunstancias ${ }^{5,6}$. El sedentarismo es un serio problema de salud que va en aumento en el mundo, y se ha identificado como un factor de riesgo de alrededor de 30 enfermedades crónicas no transmisibles (ECNT)7. Además, ha sido identificado como el cuarto factor de riesgo global de mortalidad ( $6 \%$ de las muertes a nivel mundial). Aproximadamente dos millones de muertes por año son atribuibles al sedentarismo en todo el mundo, con más del $80 \%$ de muertes por enfermedades crónicas en los países en desarrollo ${ }^{8}$.

Como parte del tratamiento de varias ECNT, se recomienda la realización de ejercicio; sin embargo, existen varias barreras para poder iniciar un programa de ejercicio, como la falta de tiempo, la falta de dinero, las situaciones climáticas, etc. Por lo tanto, el enfoque actual debe tener como prioridad el cambio del estilo de vida y la reducción del sedentarismo, conceptualizado como un proceso inicial mientras se van eliminando las barreras para la realización de ejercicio.

Se ha observado que la cantidad y el tipo de trabajo en los PS han condicionado a que permanezcan demasiado tiempo sentados, a pesar de que son conscientes de los riesgos que conlleva un estilo de vida sedentario ${ }^{9,10}$. El propósito de un estilo de vida saludable debe ser el mismo en los PS que en los 
pacientes, ya que se ha observado que las prácticas de salud en PS se relacionan positivamente con el comportamiento en los pacientes ${ }^{11}$.

En un estudio inglés se reportó que la barrera más frecuente para tener una AF adecuada en PS fue la falta de tiempo. Esta barrera coincide con la más común de la población mexicana según la Encuesta Nacional de Salud y Nutrición (ENSANUT) ${ }^{12}$.

Los objetivos de este estudio fueron conocer la cantidad de pasos al día realizados por los PS y averiguar si llevar un estilo de vida activo modifica la composición corporal.

\section{METODOLOGÍA Y PARTICIPANTES}

Se invitó a 45 PS (médicos, psicólogos, nutriólogos, optometristas) a participar en el estudio. De ellos, $33(73.3 \%)$ realizaron $<10,000$ pasos/semana en la primera fase. En la figura 1 se muestra el flujograma de pacientes. Inicialmente se les aplicó el IPAQ para evaluar el nivel de AF y se les realizó la siguiente pregunta: «¿Cuántos pasos camina al día?». A cada participante se le entregó un podómetro al inicio del estudio durante una semana. Después de esa semana se revisó el número de pasos realizados y se incluyeron en el estudio aquellos PS que registraran < 10,000 pasos al día. Se excluyeron siete sujetos por no utilizar el podómetro durante tres días consecutivos. En los 26 sujetos restantes se analizaron registros de IMC, SLM y masa muscular en kilos mediante impedancia bioeléctrica (analizador de composición corporal JAWON médico ioi353). Estos parámetros se registraron al principio, a las ocho semanas y al final del estudio.

Este estudio fue aprobado por el Comité de Ética e Investigación del Instituto Nacional de Ciencias Médicas y Nutrición Salvador Zubirán, con número

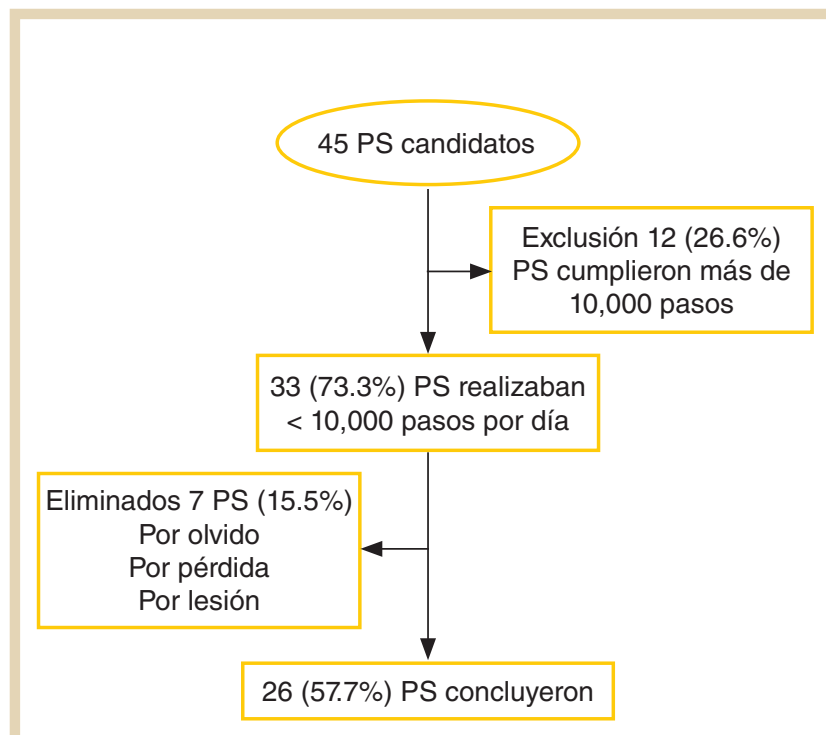

Figura 1. Flujograma de pacientes incluidos en el análisis.

1,336. Todos los participantes firmaron un consentimiento informado antes de iniciar su participación.

\section{PRESCRIPCIÓN DE ACTIVIDAD FÍSICA}

Se pidió a los participantes que cuantificaran la AF mediante el uso del podómetro modelo Fitbit ${ }^{\circledR}$ por siete días. Después de una semana se corroboró que fueran candidatos aptos para el estudio. Se obtuvo el promedio de pasos por día y por semana. Únicamente se contabilizó la AF y se les indicó retirar el podómetro en caso de realizar ejercicio.

A los PS que realizaban < 10,000 pasos al día se les indicaron rutinas específicas, como utilizar menos el carro, realizar las tareas ellos mismos y evitar estar más de 30 min seguidos sentados, para lograr la meta de 10,000 pasos durante 12 semanas. Durante cada semana registramos el número de pasos por participante hasta el final del estudio. 


\section{ANÁLISIS ESTADÍSTICO}

Para este estudio piloto se incluyeron 26 participantes. Los datos fueron analizados mediante el software de IBM SPSS versión 22. Se realizó un análisis descriptivo de pasos y composición corporal. Los porcentajes se utilizaron para valores discretos. Para detectar los cambios entre el principio y el final de las 12 semanas se realizaron las pruebas $T$ de Student para muestras relacionadas o la U de MannWhitney según correspondiera. Se consideró como significativa una $\mathrm{p}<0.05$.

\section{RESULTADOS}

De los 26 PS incluidos en este estudio, el 73\% eran mujeres y la media de edad fue de $31 \pm 11$ años. Entre las comorbilidades presentes, únicamente un $23.1 \%$ tenía sobrepeso y un $1 \%$ obesidad de grado I. No se reportó ningún otra comorbilidad.

En el IPAQ que se aplicó al principio del estudio, los participantes refirieron realizar 1.3 días (0-5) de AF a la semana, con una mediana de $83.8 \mathrm{~min}$ (0-1,200). En cuanto al ejercicio, reportaron realizar 1.7 días (0-5) a la semana, con una duración de $121 \mathrm{~min}(0-600)$. Asimismo, se reportó una mediana de horas sentados de $6.8 \mathrm{~h}(1-14)$ y de $6.2 \mathrm{~h}(5-8)$ para dormir.

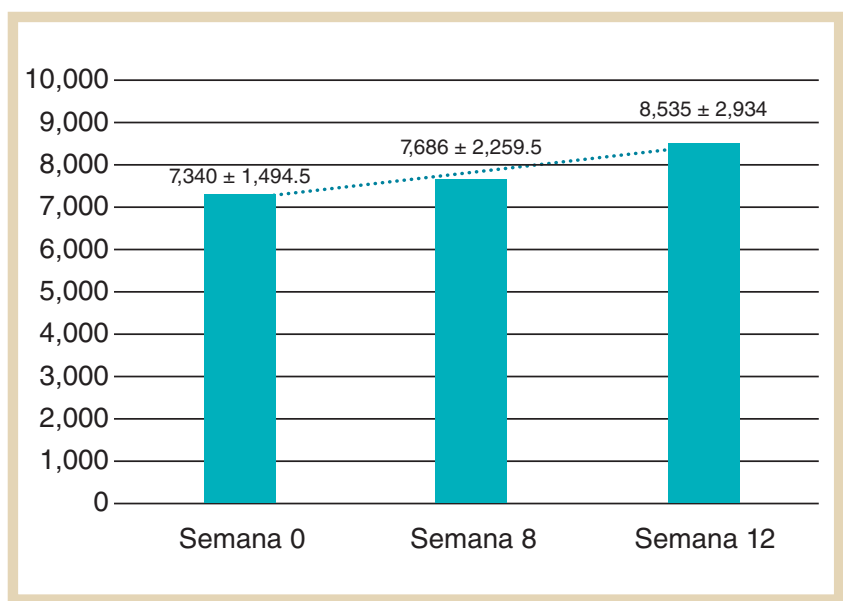

Figura 2. Promedio de pasos en las semanas 1,8 y 12.

El promedio de pasos fue incrementándose con el paso de las semanas, como se puede observar en la figura 2. Al comienzo del estudio el promedio de pasos era de 7,340 $\pm 1,494$. En la semana 12 incrementó a 8,535 $\pm 2,934(p=0.06)$. Para la semana 8 , el $15.4 \%$ de los participantes alcanzaron la meta de 10,000 pasos, y para la semana 12 este porcentaje disminuyó al 3.8\%, ya que en general aumentaron los pasos, pero no todos llegaron a la meta de 10,000 pasos diarios.

Con respecto a la bioimpedancia, se observó una tendencia en el peso de $61.6 \mathrm{~kg}$ en la semana $1 \mathrm{a}$ $61.3 \mathrm{~kg}$ en la semana $12(\mathrm{p}=0.07)$. En cuanto al IMC, MBF y SLM, no se observaron cambios significativos (Tabla 1).

TABLA 1. Cambios en la composición corporal en las semanas 1, 8 y 12

\begin{tabular}{|c|c|c|c|c|}
\hline & Semana 1 & Semana 8 & Semana 12 & $\mathrm{p}$ \\
\hline Pasos & $7,340 \pm 1,494$ & $7,686.8 \pm 2,259$ & $8,535 \pm 2,934$ & 0.06 \\
\hline Peso (kg) & $61.6 \pm 12.13$ & $61 \pm 11.7$ & $61.3 \pm 11.7$ & 0.07 \\
\hline IMC $\left(\mathrm{kg} / \mathrm{m}^{2}\right)$ & $23 \pm 3.6$ & $22.9 \pm 3.4$ & $22.9 \pm 3.4$ & 0.13 \\
\hline MBF (kg) & $18 \pm 6.5$ & $18 \pm 6.3$ & $17.9 \pm 6.4$ & 0.1 \\
\hline SLM (kg) & $40 \pm 7.2$ & $40 \pm 7.2$ & $40 \pm 7.1$ & 0.96 \\
\hline
\end{tabular}


TABLA 2. Barreras reportadas por los PS para alcanzar y mantener la meta de 10,000 pasos a la semana

\begin{tabular}{|l|c|}
\hline Barrera & $\%$ \\
\hline Ninguna & 15.4 \\
\hline Falta de tiempo & 26.9 \\
\hline Estuvo en descanso & 19.2 \\
\hline Por el tipo de trabajo & 26.9 \\
\hline Olvido del podómetro & 7.7 \\
\hline Se quedó sin pila & 3.8 \\
\hline
\end{tabular}

Se preguntó a los participantes sobre las barreras que presentaron para alcanzar y mantener la meta de 10,000 pasos al día. Las principales barreras fueron la falta de tiempo y dificultad en el lugar de trabajo, encontrándose ambas en el $26.9 \%$ de los encuestados. En la tabla 2 se muestran las principales barreras que se reportaron.

\section{DISCUSIÓN}

En el presente trabajo se evalúo el cumplimiento de las metas de AF en PS que frecuentemente prescriben ejercicio como parte del tratamiento de ECNT. Lamentablemente, en ellos mismos no se cumplen dichas metas. En un estudio similar realizado en Nigeria, se reportó que la media de pasos en PS fue de 7,270 pasos por día y el 34.4\% del personal tenía bajo nivel de $\mathrm{AF}^{8}$.

En un estudio realizado por Chan, et al. se detectó que el $65 \%$ de los PS que contabilizaron sus pasos con un podómetro alcanzaron la meta de 10,000 pasos diarios. En nuestro trabajo sólo el 15 y 3.8\% de los participantes alcanzaron la meta a las $8 \mathrm{y}$ 12 semanas, respectivamente. Ese estudio fue realizado en Nueva Zelanda, donde se le da mucha importancia a un estilo de vida saludable. Sin embargo, dado que los PS son los principales en indicar cambios en el estilo de vida, es importante dar ejemplo logrando los mismos cambios ${ }^{11}$.

Din, et al. estudiaron la percepción de los PS y su papel en la promoción de la $A F$, que describieron como un punto importante; sin embargo, en la consulta se daba más importancia a otros aspectos. En este estudio, las principales barreras fueron la distancia para ir a algún lugar a hacer ejercicio. En ese trabajo se sugiere que es importante que los PS consideren el tiempo y lugar disponibles para poder recomendar la AF en la atención primaria. En nuestro trabajo también se encontró que el tiempo era una de las principales barreras. Aunque el trabajo de Din, et al. recomienda preguntar sobre la disponibilidad de un lugar para realizar ejercicio, nosotros encontramos que la otra barrera frecuente era no poder realizarlo en el lugar de trabajo. Esto último es muy importante, ya que en muchas ocasiones se pueden sugerir al paciente distintas estrategias para aumentar su AF, incluso en la oficina o lugares de trabajo ${ }^{13}$.

El estudio de Stuij se basa en entrevistas en profundidad a 24 profesionales holandeses que brindan atención a personas con diabetes de tipo 2. Se les pidió que relataran sus experiencias con la $\mathrm{AF}$ en diferentes roles, tanto en su vida profesional como en la personal. Este enfoque narrativo se usó para explorar cómo los profesionales dieron sentido a sus experiencias con el asesoramiento de AF. Los PS que proporcionan asesoramiento de AF a las personas con diabetes de tipo 2 deben navegar entre las distintas posibilidades dentro del marco de atención de la diabetes, las opciones para integrar el AP en el mundo de la vida del paciente y las opiniones y experiencias de los profesionales con AF y vida sana ${ }^{14}$.

Crisford, et al. realizaron una revisión sistemática con estudios que incluyeron PS que realizaban 
ejercicio. Se incluyeron 30 estudios con 7,734 PS no médicos. La autoeficacia en la promoción de la AP, las creencias positivas en los beneficios de la AF, la evaluación de la AF de los pacientes y la capacitación para la promoción de AF fueron los principales factores asociados con la participación en la promoción de AF. Los factores identificados pueden ser útiles para guiar el desarrollo de estrategias para alentar una mayor participación de los PS en la promoción de la $\mathrm{AF}^{15}$.

Alghafri, et al. realizaron un estudio cualitativo con el objetivo de determinar la percepción de los PS de las barreras y oportunidades, las responsabilidades del personal y los enfoques promocionales plausibles de la AP. Otras barreras que se reportaron en este trabajo fueron la falta de guías para indicar AF, situaciones sociales (trabajo) y falta de facilidades para realizarlo ${ }^{16}$.

Otro dato que llama la atención es el entusiasmo inicial y la disminución de este al final de las 12 semanas. Este comportamiento es frecuente en los cambios que se requieren para el manejo de enfermedades crónicas cuando no se abordan estrategias motivacionales para el mantenimiento a largo plazo, si no hay un recordatorio o un estímulo que ayude a mantener la meta ${ }^{1}$. Esto depende también de factores culturales y educativos, como demostraron Chan, et al. en Nueva Zelanda, quienes reportaron que el promedio de pasos en PS fue de 10,620 pasos por día y el $65 \%$ de ellos alcanzaron la meta ${ }^{11}$.

La población incluida en el estudio son los primeros que necesitan saber y enseñar respecto al incremento de AF. Una de las premisas importantes es enseñar con el ejemplo y no pedir al paciente algo que los mismos PS no realizamos. Analizar en los propios PS cuáles son los factores que impiden llevar cambios nos puede llevar a generar mayor empatía para comunicar las estrategias y soluciones a las barreras que se presenten para tener un estilo de vida más saludable.

Aunque éste fue un estudio piloto, encontramos una diferencia en los niveles de AF. Por otro lado, el tamaño de la muestra no fue suficiente para demostrar el efecto a largo plazo y los cambios en los parámetros clínicos.

\section{DECLARACIÓN DE CONFLICTO DE INTERÉS}

Los autores de este artículo no reportan conflictos de interés.

\section{GRUPO DE TRABAJO CAIPADI}

Denise Arcila-Martínez, Rodrigo Arizmendi-Rodríguez, Oswaldo Briseño-González, Humberto Del Valle-Ramírez, Arturo Flores-García, Eduardo González-Flores, Fernanda Garnica-Carrillo, Mariana Granados-Arcos, Héctor Infanzón-Talango, María Victoria Landa-Anell, Claudia Lechuga-Fonseca, Arely López-Reyes, Marco Antonio Melgarejo-Hernández, Angélica Palacios-Vargas, Eder Patiño-Rivera, Liliana Pérez Peralta, David Rivera de la Parra, Francis Rojas-Torres, Marcela Ruiz-Cervantes, Vanessa Ruiz-González, Sandra Sainos-Muñoz, Alejandra Sierra-Esquivel, Erendi Tinoco-Ventura, María Luisa Velasco-Pérez, Héctor Velázquez-Jurado y Verónica Zurita-Cortés.

\section{BIBLIOGRAFÍA}

1. Colberg S, Sigal R, Yardley J, Riddell M, Dunstan D, Dempsey P, et al. Physical Activity/Exercise and Diabetes: A Position Statement of the American Diabetes Association. Diabetes Care. 2016;39(11):2065-79.

2. Tudor-Locke C, Craig CL, Thyfault JP, Spence JC. A step-defined sedentary lifestyle index : <5000 steps/day. Appl Physiol Nutr Metab. 2013;38(2):100-14. 
3. Ainsworth B, Cahalin L, Buman M, Ross R. The current state of physical activity assessment tools. Prog Cadiovasc Dis. 2015;57(4):387-95.

4. Blake H, Mo PK, Lee S, Batt ME. Health in the NHS: lifestyle behaviours of hospital employees. Perspect Public Health. 2012;132(5):213-5.

5. Henson J, Dunstan DW, Davies MJ, Yates T. Sedentary behaviour as a new behavioural target in the prevention and treatment of type 2 diabetes. Diabetes Metab Res Rev. 2016;32 Suppl 1:213-20.

6. McKay J, Wright A, Lowry R, Steele K, Ryde G, Mutrie N. Walking on prescription: The utility of a pedometer pack for increasing physical activity in primary care. Patient Educ Couns. 2009;76(1):71-6.

7. Levine JA. Sick of sitting. Diabetologia. 2015;58(8):1751-8.

8. Owoeye O, Tomori A, Akinbo S. Pedometer-determined physical activity profile of healthcare professionals in a Nigerian tertiary hospital. Niger Med J. 2016;57(2):99-103.

9. Ching P, Willet W, Rimm E, Colditz G, Gortmaker S, Stampfer M. Activity level and risk of overweight in male health professionals. Am J Public Health. 1996;86(1):25-30.

10. Dasgupta K, Rosenberg E, Joseph L, Cooke AB, Trudeau L, Bacon SL, et al. Physician step prescription and monitoring to improve Arterial health (SMARTER): A randomized controlled trial in patients with type 2 diabetes and hypertension. Diabetes Obes Metab. 2017;19(5): 695-704.
11. Chan L, McNaughton H, Weatherall M. Are physical activity levels of health care professionals consistent with activity guidelines? A prospective cohort in New Zealand. JRSM Cardiovasc Dis. 2018;7: 2048004017749015.

12. Instituto Nacional de Salud Pública. Encuesta de salud y nutrición de Medio Camino. 2016. Informe final de resultados. Disponible en: https:// www.gob.mx/cms/uploads/attachment/file/209093/ENSANUT.pdf

13. Din NU, Moore GF, Murphy S, Wilkinson C, Williams NH. Health professionals' perspectives on exercise referral and physical activity promotion in primary care: Findings from a process evaluation of the National Exercise Referral Scheme in Wales. Health Educ J. 2015;74(6): 743-57.

14. Stuij M. 'Physical activity, that's a tricky subject.' Experiences of health care professionals with physical activity in type 2 diabetes care. BMC Health Serv Res. 2018;18(1):297.

15. Crisford P, Winzenberg T, Venn A, Schultz M, Aitken D, Cleland V. Factors associated with physical activity promotion by allied and other non-medical health professionals. Patient Educ Couns. 2018. [Epub ahead of print]

16. Alghafri TS, Alharthi SM, Al-Balushi S, Al-Farsi Y, Al-Busaidi Z, Bannerman $\mathrm{E}$, et al. Health professionals' perceptions about physical activity promotion in diabetes care within primary health care settings in Oman. Heliyon. 2018;3(12):e00495. 